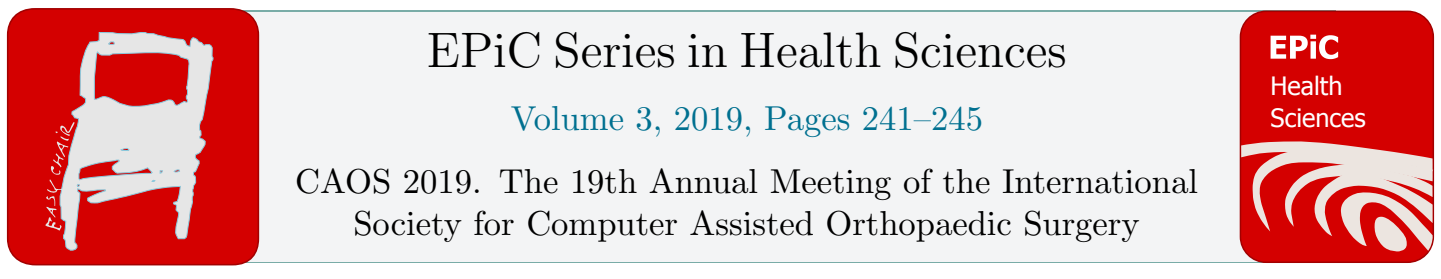

\title{
1-Year Patient Outcomes for Robotic-Arm Assisted vs. Manual Total Knee Arthroplasty
}

\author{
Robert Marchand ${ }^{1}$; Nipun Sodhi ${ }^{2}$ Hiba K. Anis 3 ; Joseph O. Ehiorobo ${ }^{2}$; Jared M. \\ Newman ${ }^{4}$; Kevin Marchand ${ }^{1}$; Kelly Taylor ${ }^{1}$; Caitlin Condrey ${ }^{1}$; Matthew S. \\ Hepinstall $^{2}$; Michael A. Mont ${ }^{2}$ \\ ${ }^{1}$ Ortho Rhode Island, Wakefield, Rhode Island \\ ${ }^{2}$ Lenox Hill Hospital, Northwell Health, New York, New York \\ ${ }^{3}$ Cleveland Clinic, Cleveland, Ohio \\ ${ }^{4}$ SUNY Downstate, Brooklyn, New York
}

\section{Introduction:}

Robotic-arm assisted (RAA) total knee arthroplasty (TKA) has been shown to potentially have certain pre- and intra-operative advantages over manual techniques ${ }^{1,2}$. Although there are many studies on the potential alignment advantages when using the RAA system, there have been questions regarding patient reported outcomes. To the best of the author's knowledge, there has only been one reported study on roboticarm assisted patient satisfaction ${ }^{3}$. This study compared 6-month WOMAC scores of a consecutive series of 20 RAA TKAs to 20 manual TKAs performed by a single surgeon based on. The authors found significant differences in the RAA cohort for WOMAC pain and total scores when compared to the manual cohort $(\mathrm{p}<0.05)$. Mean physical function score was also found to be better for the RAA cohort $(p=0.055)$. Although this study identified more favorable patient satisfaction outcomes in the robotic-arm assisted cohort, further studies at longer term follow up and with greater sample sizes are needed to substantiate these results. Therefore, the purpose of this study was to use this index in order to compare: 1) total; 2) physical function; and 3) pain scores for manual vs. RAA patients. 


\section{Methods:}

Robotic-arm assisted total knee arthroplasties were performed by a single orthopaedic surgeon at a high volume institution. After allowing for an appropriate learning curve window (14 RAA TKAs), cases from an initial 6-month period, between September 16, 2016 and March 16, 2017, were evaluated. During this 6-month window, a total of 153 RAA TKAs were performed. Of the 153 patients, 53 completed their 1-year WOMAC survey, and were included for analysis. A total of 53 consecutive manual TKAs performed by the same surgeon between April 21 $1^{\text {st }}, 2015$ and December $15^{\text {th }}$, 2015 were used for comparison.

The Western Ontario and McMaster Universities Arthritis (WOMAC) Index is a validated hip and knee survey ${ }^{4-7}$. It is a self-administered survey, that consists of 24 questions distributed between 3 subcategories: pain (5 questions), stiffness (2 questions), and physical function (18 questions). Higher WOMAC scores correlate to worse total mobility and function. Both the manual and RAA cohort patients completed these surveys at their pre-operative and 1 year post-operative visits.

On univariate analysis, there were no significant differences in pre-operative WOMAC total, physical function, and pain scores between the two cohorts.

Additionally, no significant differences in patient demographics were found between the two cohorts.

Baseline patient demographics (age, sex, and body mass index) as well as WOMAC scores were compared between the two cohorts with univariate analyses.

Independent samples t-tests were performed for continuous variables to compare means and Pearson's chi-squared tests were performed for categorical variables. Histograms were created in order to compare the distribution of total, function, and pain WOMAC scores between the RAA and manual cohorts

Multicollinearity between the study variables were tested with variance inflation factors (VIFs); VIFs greater than 3 identified strong collinearity. No collinearity was identified between the study covariates; therefore, they were all included in the multivariate regression analyses. Multivariate models with stepwise backward linear regression were utilized in order to further evaluate the associations of surgical technique with pain, function, and total WOMAC scores. The study covariates analyzed were: surgical technique (manual vs. RAA), age, sex, and BMI. All of these variables were included in the regression model, and variables with the weakest correlations were sequentially removed in subsequent models until that with the strongest association was identified.

All data analyses were performed using SPSS version 22.0 (International Business Machine Corporation, Armonk, New York) and statistical significance was maintained at a p-value of less than 0.05 . 


\section{Results:}

\section{Total WOMAC Scores}

1-year post-operative total WOMAC scores were significantly lower in the roboticarm assisted cohort $(\mathrm{p}<0.05)$ compared to the manual cohort, indicating overall improved outcomes for RAA patients. The mean total WOMAC score for the manual cohort was $9 \pm 8$ points (range, 0 to 27 points), while the mean total score for the RAA cohort was $6 \pm 6$ points (range, 0 to 29 points) (Table 1).

On backward regression analyses RAA was found to be significantly associated with lower total WOMAC scores (beta coefficient [ $\beta$ ] -0.208, SE [standard error] 1.401, $\mathrm{p}<0.05$ ) at 1-year post-operatively. Specifically, in each stepwise model, use of the RAA surgical technique was found to have the strongest association with total WOMAC scores when compared to age, gender, and BMI (Table 2).

\section{Physical Function Scores}

For the manual cohort, the mean physical function score was $6 \pm 5$ points (range, 0 to 16 points), whereas the mean physical function score for the RAA cohort was $4 \pm 4$ points (range, 0 to 15 points; $p<0.05$ ), indicating better physical function outcomes for RAA patients.

Multivariate backward regression also found the use of RAA TKA to have a significant association $(\beta-0.216$, SE $0.829, \mathrm{p}<0.05)$ with lower and therefore improved function scores 1 year post-operatively. Specifically, in each stepwise model, use of the RAA surgical technique was found to have the strongest association with WOMAC function scores when compared to age, gender, and BMI.

\section{Pain Scores}

The mean post-operative pain score for the manual cohort was found to be $3 \pm 4$ points (range, 0 to 11 points), whereas the mean post-operative pain score for the RAA cohort was found to be lower at $2 \pm 3$ points (range, 0 to 14 points; $p=0.06$ ).

Of all the study covariates included in the model, the use of RAA had the strongest association $(\beta-0.181$, SE $0.623, p=0.063)$ with lower pain scores. Specifically, in each stepwise model, use of the RAA surgical technique was found to have the strongest association with WOMAC pain scores when compared to age, gender, and BMI.

\section{Table 1. 1-year Post-Operative WOMAC Scores}




\begin{tabular}{|c|c|c|c|c|c|}
\hline \multirow{2}{*}{$\begin{array}{l}\text { 1-year Post- } \\
\text { Operative } \\
\text { WOMAC Scores }\end{array}$} & \multicolumn{2}{|c|}{ Manual TKA } & \multicolumn{2}{|c|}{$\begin{array}{c}\text { Robotic-Assisted } \\
\text { TKA }\end{array}$} & \multirow[t]{2}{*}{$\begin{array}{c}\text { p- } \\
\text { value }\end{array}$} \\
\hline & $\begin{array}{c}\text { Mean } \pm \\
\text { SD }\end{array}$ & Range & $\begin{array}{c}\text { Mean } \pm \\
\text { SD }\end{array}$ & Range & \\
\hline Total & $9 \pm 8$ & & $6 \pm 6$ & & $<0.05$ \\
\hline Physical Function & $6 \pm 5$ & 0 to 16 & $4 \pm 4$ & 0 to 15 & $<0.05$ \\
\hline Pain & $3 \pm 4$ & 0 to 11 & $2 \pm 3$ & 0 to 14 & 0.06 \\
\hline
\end{tabular}

p-values: independent samples t-test

Table 2. Association Between Use of RAA Surgical Technique and WOMAC Scores

\begin{tabular}{lccc}
\hline $\begin{array}{l}\text { 1-year Post- } \\
\text { Operative }\end{array}$ & $\begin{array}{c}\text { Standardized } \\
\text { Coefficient ( } \boldsymbol{\beta})\end{array}$ & $\begin{array}{c}\text { Standard Error } \\
\text { (SE) }\end{array}$ & $\begin{array}{c}\text { p- } \\
\text { value }\end{array}$ \\
\hline \hline Total & -0.208 & 1.401 & $<\mathbf{0 . 0 5}$ \\
Physical Function & -0.216 & 0.829 & $<\mathbf{0 . 0 5}$ \\
Pain & -0.181 & 0.623 & 0.063 \\
\hline
\end{tabular}

\section{Conclusion:}

With newer surgical technologies constantly being introduced, it is imperative to continue to evaluate these new modalities, particularly in their abilities to improve patient satisfaction outcomes ${ }^{8-11}$. To the best of our knowledge, this is one of the first study evaluating 1 year patient satisfaction outcomes utilizing this new robotic-arm assisted total knee arthroplasty ${ }^{12}$. The 1 year post-operative patient satisfaction results from this study corroborate those of an earlier report on 6-month outcomes. Based on these combined outcomes, patient can expect superior early satisfaction from roboticarm assisted TKA.

\section{References}

1. Marchand RC, Sodhi N, Khlopas A, Sultan AA, Higuera CA, Stearns KL, et al. Coronal Correction for Severe Deformity Using Robotic-Assisted Total Knee Arthroplasty. J Knee Surg Germany, 2018;31(1):2-5.

\section{Hampp EL, Chughtai M, Scholl LY, Sodhi N, Bhowmik-Stoker M,}


Jacofsky DJ, et al. Robotic-Arm Assisted Total Knee Arthroplasty

Demonstrated Greater Accuracy and Precision to Plan Compared with Manual Techniques. J Knee Surg Germany, 2018;

3. Marchand RC, Sodhi N, Khlopas A, Sultan AA, Harwin SF, Malkani AL, et al. Patient Satisfaction Outcomes after Robotic Arm-Assisted Total Knee Arthroplasty: A Short-Term Evaluation. J Knee Surg 2017;

4. Jauregui JJ, Banerjee S, Cherian JJ, EImallah RDK, Mont MA. Rating Systems to Assess the Outcomes After Total Knee Arthroplasty. Surg Technol Int United States, 2015;26:289-294.

5. Pierce TP, EImallah RDK, Cherian JJ, Jauregui JJ, Mont MA.

Standardized Questionnaire Time Burden for Practitioners and Patients. Surg Technol Int United States, 2015;26:302-306.

6. Chughtai M, Khlopas A, Thomas M, Gwam CU, Jauregui JJ, EImallah RK, et al. Development of an Encompassing Questionnaire for Evaluating the Outcomes Following Total Knee Arthroplasty. Surg Technol Int United States, 2017;30:306-313.

7. Chughtai M, Khlopas A, Mistry JB, Gwam CU, Elmallah RK, Mont MA. Time Burden of Standardized Hip Questionnaires. Surg Technol Int United States, 2016;28:280-284.

8. Schiraldi M, Bonzanini G, Chirillo D, Tullio V de. Mechanical and kinematic alignment in total knee arthroplasty. Ann Transl Med [Internet] AME Publishing Company, 2016;4(7):130.

9. Cherian JJ, Kapadia BH, Banerjee S, Jauregui JJ, Issa K, Mont MA. Mechanical, Anatomical, and Kinematic Axis in TKA: Concepts and Practical Applications. Curr Rev Musculoskelet Med [Internet] Boston: Springer US, 2014;7(2):89-95.

10. Berend ME, Ritter MA, Meding JB, Faris PM, Keating EM, Redelman R, et al. Tibial component failure mechanisms in total knee arthroplasty. Clin Orthop Relat Res United States, 2004;(428):26-34.

11. Sikorski JM. Alignment in total knee replacement. J Bone Joint Surg Br England, 2008;90(9):1121-1127.

12. Kayani B, Konan S, Tahmassebi J, Pietrzak JRT, Haddad FS. Roboticarm assisted total knee arthroplasty is associated with improved early functional recovery and reduced time to hospital discharge compared with conventional jig-based total knee arthroplasty. Bone Joint J England, 2018;100-B(7):930-937. 